\title{
INFLUENCE DE LA TEMPÉRATURE SUR LA PRODUCTION PISCICOLE EN ÉTANG
}

\author{
par J. A. TIMMERMANS \\ Ingénieur des Eaux et Forêts. \\ Station de Recherches des Eaux et Forêts (Groenendaal-Belgique).
}

I a production piscicole des étangs dépend de beaucoup de facteurs. Parmi les plus importants il faut mentionner la qualité de l'eau et du fond de l'ètang, le climat, le mode d'exploitation. L.'interaction de ces facteurs est complexe et difficile à saisir.

L'influence de la qualité de l'eau et du fond est relativement bien connue et constitue, la plupart du temps, la base pour apprécier la productivité des étangs dans une région donnée. Le mode d'exploitation a une importance non négligeable sur la production : l'espèce ou le mélange d'espèces qu'on élève, l'âge ou la taille des poissons, la densité de la mise en charge influencent la production. Le climat aussi est un facteur important : la température moyenne et les extrêmes, le rayonnement, la durée de la bonne saison. Pour une espèce donnée, plus la température de l'eau approche de la température optima de croissance et plus la durée de cette température optima est longue, plus la production annuelle sera grande, tous les autres facteurs restant constants. C'est ainsi que la production annuelle par unité de surface dans les étangs où l'on élève la Carpe, poisson préférant une eau chaude (20 à $25^{\circ}$ C) et élevé à des latitudes fort différentes, augmente des régions tempérées vers les régions subtropicales et tropicales.

Mais aussi la production piscicole peut varier considérablement d'une année à une autre dans une même région à cause des variations climatologiques et spécialement à cause de la température. Nous avons pu le constater en Belgique pour la Carpe pendant sa première année d'élevage.

Durant six années consécutives, de 1951 a 1959 , le contrôle de différents étangs a pu être fait à la pisciculture de Limal, aussi bien pour l'élevage de carpillons de six semaines que pour l'élevage de carpillons d'un été, avec et sans alimentation artificielle. Certaines de ces observations ont fait l'objet de publications antérieures (HCE' et Tim- 
Mermans, 1955, 1956, 1957). La production moyenne des étangs contròlés a varié d'une année à l'autre et il y a une relation certaine entre cette production et la températưre. Bien que le but de ce contrôle ne fut pas de connaître cette relation, il nous a semblé intéressant de la faire apparaître sur des graphiques $(2,3,4)$. La production moyenne journalière en kilogrammes par hectare y figure en ordonnée, la température moyenne journalière en ${ }^{\circ} \mathrm{C}$ en abcisse.

Les étangs dans lesquels les contrôles ont été faits sont de petits étangs rectangulaires et contigus, d'une superficie individuelle d'environ 3,5 ares. L'eau d'alimentation est alcaline : $p \mathrm{H}$ de 7,5 et SBV de 2,7.

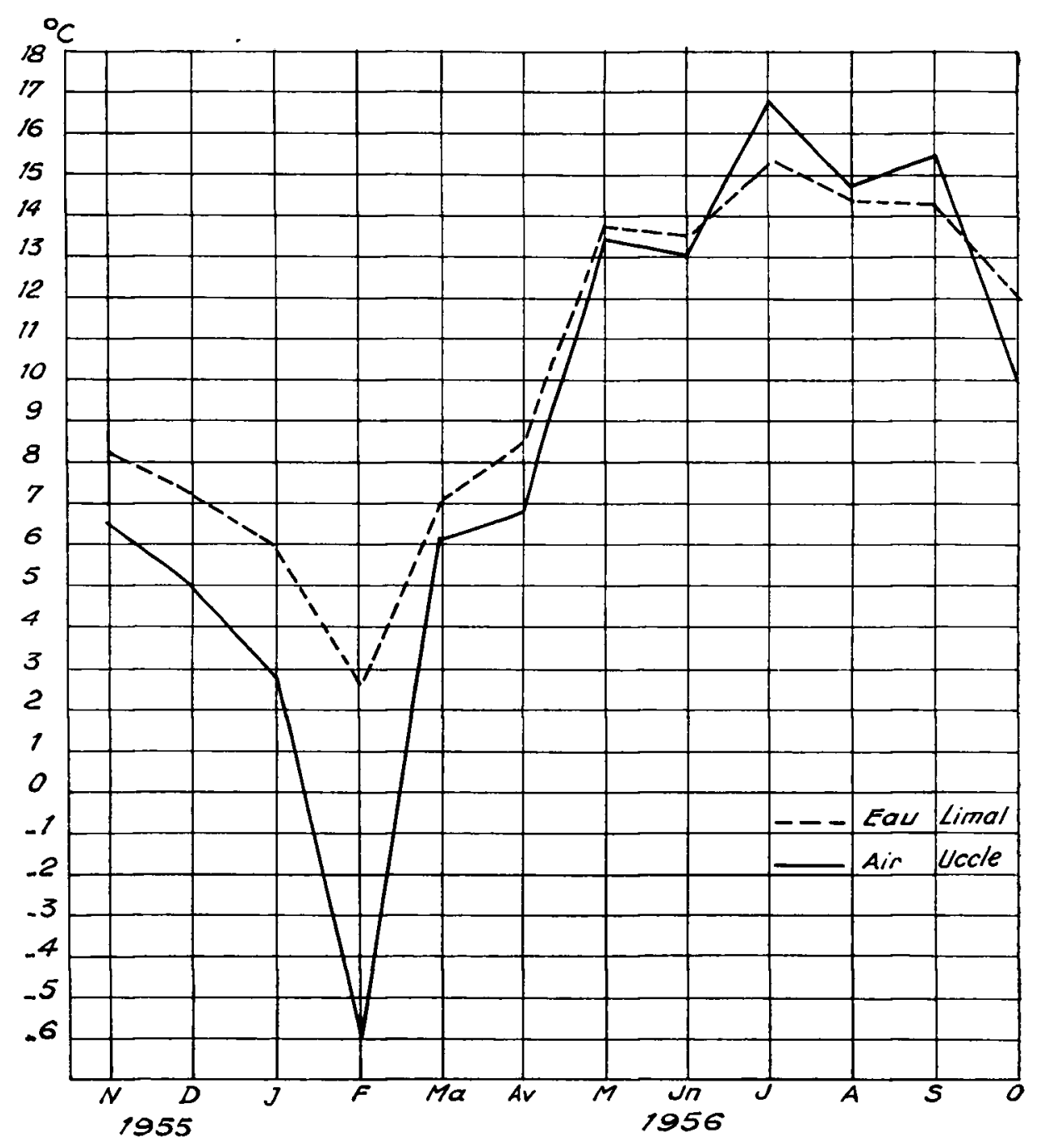

Fig. 1. - Evolution de la température moyenne mensuelle du ruisseau d'alimentation des étangs a la pisciculture de Limal et de la température moyenne mensuelle de l'aí à Uccle. 


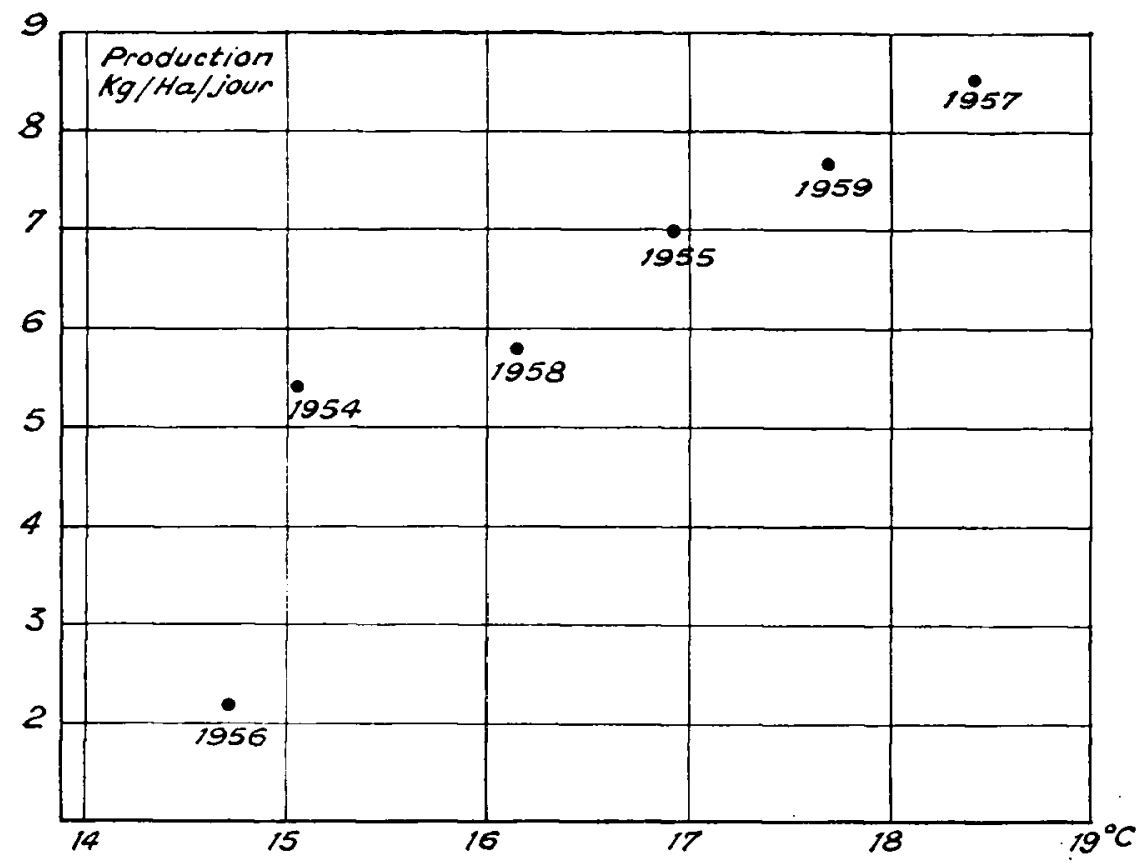

Fig. 2. - Carpicullure, étangs de premier alevinage.

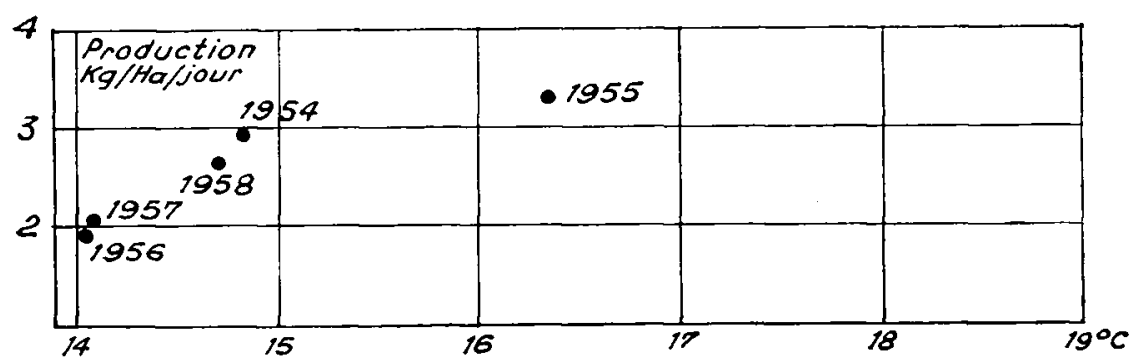

Fig. 3. - Carpiculture, étangs de second alevinage sans alimentation artificielle.

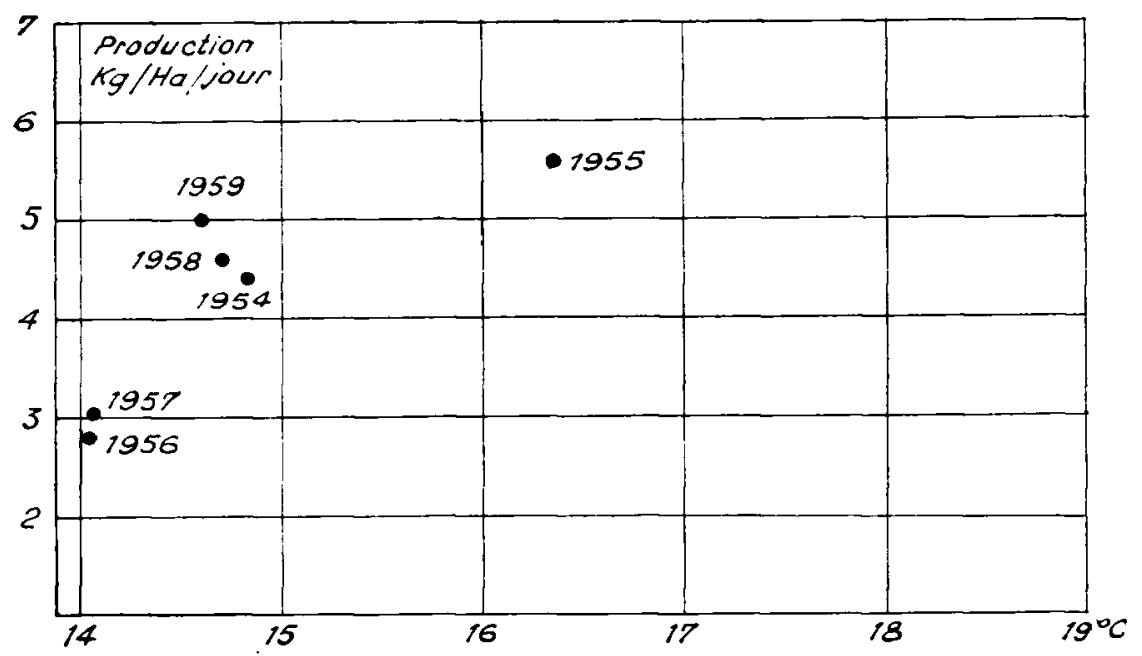

Fig. 4. - Carpiculture, étangs de second alprinage avec alimentation artificielle. 
Les productions moyennes journalières durant les différentes années qui figurent sur les graphiques ont été calculees de la façon suivante. Pour chaque étang, la production à l'hectare a été déterminée durant la période d'élevage envisagée et cette production a été divisée par le nombre de jours qu'a duré cet élevage, soit le premier alevinage, soit le second alevinage sans alimentation artificielle, soit le second alevinage avec alimentation artificielle. Les moyennes de ces résultats pour chaque année et pour chaque élevage envisagés figurent sur les graphiques.

La température qui a influencé la production des étangs est certainement celle de l'eau des étangs. Cette température n'a pas été relevée systématiquement. Les températures moyennes journalières qui figurent sur les graphiques sont les températures moyennes de l'air à Uccle, d'après les données de l'Institut Royal Météorologique. Ce sont les moyennes de 13 mesures bihoraires de 0 à 24 heures, données pour les trois décades de chaque mois. Nous avons calculé pour les différentes années la température moyenne journalière de chaque période d'élevage envisagée.

La distance entre Limal, où sont situés les étangs contrôlés et Cccle, où les températures de l'air ont été prises, est de $20 \mathrm{~km}$. Limal se trouve à une altitude de $55 \mathrm{~m}$., Uccle à une altitude de $100 \mathrm{~m}$.

Le graphique I donne la comparaison entre la température moyenne mensuelle de l'air prise à Uccle et la température moyenne mensuelle de l'eau du ruisseau d'alimentation à Limal, de Novembre 1955 à Octobre 1956. On constate que la température de l'eau à Limal évolue comme la température de l'air à Uccle, mais que l'amplitude est moins prononcée pour la température de l'eau.

Pour déterminer l'influence de la température de façon plus exacte, il eut fallu que les facteurs autres que les facteurs climatologiques aient été identiques durant les six années d'observation. Ceci n'a pas été le cas et parmi une série de 10 étangs, le nombre d'étangs contrôlés et leur mise en charge ont varié. Le nombre d'étangs contrôlés est indiqué ci-après. Entre parenthèses figure le nombre moyen de poissons récoltés au mètre carré.

\begin{tabular}{|c|c|c|c|}
\hline Année & $\begin{array}{c}\text { Étangs de } \\
1^{\text {er }} \text { alevinage }\end{array}$ & $\begin{array}{c}\text { Etangs de } \\
2^{\mathrm{e}} \text { alevinage } \\
\text { sans alimentation } \\
\text { artificielle }\end{array}$ & $\begin{array}{c}\text { Etangs de } \\
2^{\mathrm{e}} \text { alevinage } \\
\text { avec alimentation } \\
\text { artificielle }\end{array}$ \\
\hline $\begin{array}{l}1954 \\
1955 \\
1956 \\
1957 \\
1958 \\
1959\end{array}$ & $\begin{array}{rr}4 & (12,9) \\
8 & (7,5) \\
4 & (5,6) \\
10 & (10,8) \\
4 & (12,1) \\
4 & (7,3)\end{array}$ & 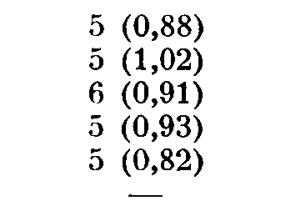 & $\begin{array}{ll}4 & (0,98) \\
4 & (1,14) \\
4 & (1,35) \\
5 & (1,85) \\
3 & (1,58) \\
4 & (1,46)\end{array}$ \\
\hline
\end{tabular}


Nonobstant ces lacunes, dues au fait que le but des contròles n'ètait pas d'établir une relation entre la température et la production, il est certain que l'on peut reconnaître que la température, plus que les autres facteurs qui ont varié, a influencé la production, comme on peut le voir sur les graphiques 2,3 et 4 .

On peut dire que plus la température moyenne journalière est élevée, plus la production journalière est élevée aussi. Ceci est surtout caractèristique pour les étangs de premier alevinage dont la période moyenne de croissance fut de 44 jours, donc un peu plus de 6 semaines. Pour l'ensemble des six années d'observations, cette période s'est étalée du 25 mai au 26 juillet. Pour les étangs de $2^{\text {e }}$ alevinage la période moyenne de croissance fut de 92 jours. Pour l'ensemble des six années d'observation cette période s'est étendue du 16 Juillet au 13 Novembre.

Il est intéressant de constater aussi que, pour une même saison, il y a des périodes à production journalières fort différentes. C'est le cas pour l'année 1957, pendant laquelle la production journalière fut excellente dans les étangs de $1^{\text {er }}$ alevinage mais médiocre pour les étangs de $2^{e}$ alevinage.

Il n'est évidemment pas possible, à l'aide des données obtenues ici, d'exprimer mathématiquement la relation entre la production et la température journalières pour l'élevage de carpillons de 6 semaines et de carpillons d'un été. Ceci serait toutefois réalisable, si l'on conduisait des essais dans ce but durant une période assez longue et dans une région où le climat peut varier considérablement d'une année à l'autre, comme c'est le cas pour une région à climat tempéré océanique.

Groenendaal, Mai 1962.

\section{BIBL IOGRAPHIE}

Bulletins mexsuels de l'institut Royal Météohologique de Belgique.

M. Huet et J. A. Timmermans : Observations concernant la production de carpillons et de jeunes brochetons. Pêche et Pisciculture, no 3 , Mars $1955 ; n^{\circ} 2$, Février $1956 ; n^{\circ} 10$, Octobre 1957. 